\title{
Analyzing Angular Distributions for Two-Step Dissociation Mechanisms in Velocity Map Imaging
}

\author{
Daniel B. Straus, ${ }^{\dagger, \S}$ Lynne M. Butler, ${ }^{\ddagger}$ Bridget W. Alligood, ${ }^{\dagger, \|}$ and Laurie J. Butler, ${ }^{*} \dagger$ \\ ${ }^{\dagger}$ Department of Chemistry and the James Franck Institute, University of Chicago, Chicago, Illinois 60637, United States \\ ${ }^{\star}$ Department of Mathematics and Statistics, Haverford College, Haverford, Pennsylvania 19041, United States
}

\section{Supporting Information}

ABSTRACT: Increasingly, velocity map imaging is becoming the method of choice to study photoinduced molecular dissociation processes. This paper introduces an algorithm to analyze the measured net speed, $P\left(v_{\text {net }}\right)$, and angular, $\beta\left(v_{\text {net }}\right)$, distributions of the products from a two-step dissociation mechanism, where the first step but not the second is induced by absorption of linearly polarized laser light. Typically, this might be the photodissociation of a $\mathrm{C}-\mathrm{X}$ bond $(\mathrm{X}=$ halogen or other atom) to produce an atom and a momentum-matched radical that has enough internal energy to subsequently dissociate (without the absorption of an additional photon). It is this second step, the dissociation of the unstable radicals, that one wishes to study, but the measured net velocity of the final products is the vector sum of the velocity imparted to the radical in the primary photodissociation (which is determined by taking data on the momentum-matched atomic cophotofragment) and the additional velocity vector imparted in the subsequent dissociation of the unstable radical. The algorithm allows one to determine, from the forward-convolution fitting of the net velocity distribution, the distribution of velocity vectors imparted in the second step of the mechanism. One can thus deduce the secondary velocity distribution, characterized by a speed distribution $P\left(v_{1,2^{\circ}}\right)$ and an angular distribution $I\left(\theta_{2^{\circ}}\right)$, where $\theta_{2^{\circ}}$ is the angle between the dissociating radical's velocity vector and the additional velocity vector imparted to the product detected from the subsequent dissociation of the radical.

\section{INTRODUCTION}

Velocity map imaging, originally introduced to study photoinduced processes, is now being applied to the study of radical intermediates in bimolecular reactions. The radical intermediate may be produced under collision-free conditions with modest to high internal energies (typically between 10 and $50 \mathrm{kcal} /$ $\mathrm{mol}$ ) by photodissociating a halogenated precursor in the main chamber of the apparatus (not in the nozzle region). The nascent internal energy distribution of the radical photoproduct, which is not cooled in the supersonic expansion, is then preserved, and the higher internal energy radicals can dissociate on portions of the ground state potential energy surface relevant to the bimolecular reaction, without the absorption of a second photon. The analysis of such two-step dissociations is routinely done for data taken in crossed lasermolecular beam scattering apparatuses using the wellestablished program CMLAB2. ${ }^{1}$ However, if such two-step dissociations are studied in a velocity map imaging apparatus, no data analysis methodology currently exists to properly analyze the net velocities measured. We have recently introduced an algorithm ${ }^{2}$ to model the net speed distribution for such a process to extract the energy partitioned to relative kinetic energy in the dissociation of the radical intermediate, but that algorithm did not treat the angular distribution of the net velocity vectors. If the initial photolysis step is anisotropic, which is the usual case, the angular distribution of the net velocity vector is affected both by this anisotropy and by the subsequent dissociation dynamics of the radical intermediate generated.
This paper describes a new method for characterizing data taken on a velocity map imaging apparatus through two-step photodissociation mechanisms. Martinez-Haya and co-workers ${ }^{3}$ developed a method to simulate the photofragment angular distribution for a two-step mechanism, but their model assumes that both the primary and secondary dissociations require the absorption of one or more photons to occur. Here we describe a method to compute the net angular distribution for the products of a two-step mechanism in which only the primary dissociation is photoinduced. Our model thus must use Euler angle rotations to analytically compute the angular distribution for the two-step dissociation mechanism.

For clarity, this paper uses the language of the two-step dissociation of chloroacetone to ketene and methyl:

$$
\begin{aligned}
& \mathrm{CH}_{3} \mathrm{C}(\mathrm{O}) \mathrm{CH}_{2} \mathrm{Cl} \stackrel{193 \mathrm{~nm}}{\longrightarrow} \mathrm{CH}_{3} \mathrm{C}(\mathrm{O}) \mathrm{CH}_{2}+\mathrm{Cl} \\
& \mathrm{CH}_{3} \mathrm{C}(\mathrm{O}) \mathrm{CH}_{2} \rightarrow \mathrm{CH}_{2} \mathrm{CO}+\mathrm{CH}_{3}
\end{aligned}
$$

This specific example is treated in the Supporting Information.

\section{EXPERIMENTAL METHODS}

The velocity map imaging technique used to collect data and the specific parameters used in our experiments have been

Special Issue: Joel M. Bowman Festschrift

Received: January 11, 2013

Revised: February 16, 2013 
described previously. ${ }^{2,4}$ The technique is briefly summarized here. A halogenated precursor molecule is seeded in an inert gas, and the gaseous mixture is expanded through a pulsed valve into a high vacuum chamber. The beam then passes through a skimmer to collimate it, and the molecules are dissociated using vertically polarized light. The resulting photofragments and any secondary dissociation products are then photoionized (at 118 $\mathrm{nm}$ for chloroacetone) after a delay of about $40 \mathrm{~ns}$. The ions travel down a grounded time-of-flight tube toward the detector, which is a microchannel plate assembly coupled to a phosphor screen. A CCD camera records images of the ions' impact positions. We process the obtained images using the ioncounting method, ${ }^{5}$ and the raw images are symmetrized about the vertical and horizontal axes in the data analysis.

\section{COMPUTATIONAL MODEL}

The $2 \mathrm{D}$ image recorded in the experiment is first processed with an inverse Abel transformation executed with the BASEX program. ${ }^{6}$ The resulting $3 \mathrm{D}$ image gives the net velocity distribution of the detected products. After a calibrated pixel-tospeed conversion, the BASEX output reports a speed distribution $P\left(v_{\text {net }}\right) \propto \int_{0}^{\pi} I\left(v_{\text {net }} \theta\right) v_{\text {net }}^{2} \sin \theta_{\text {net }} \mathrm{d} \theta_{\text {net }}$ and a speed dependent anisotropy parameter $\beta\left(v_{\text {net }}\right){ }^{7}$ Images from two-step dissociations (such as the dissociation of chloroacetone to $\mathrm{Cl}+$ $\mathrm{CH}_{3} \mathrm{C}(\mathrm{O}) \mathrm{CH}_{2}$, and the secondary dissociation of the latter to ketene and methyl) contain velocity contributions from both the primary and secondary dissociation. The speed and angular distributions of the products of the primary $\mathrm{C}-\mathrm{Cl}$ bond photofission in chloroacetone are determined by taking $\mathrm{Cl}$ data; by conservation of momentum, we can determine the velocity $\vec{v}_{1,1^{\circ}}$ imparted to $\mathrm{CH}_{3} \mathrm{C}(\mathrm{O}) \mathrm{CH}_{2}$. We then use a forward convolution method to fit the net speed distribution, $P\left(v_{\text {net }}\right)$, by iteratively manipulating the distribution of secondary speeds, $P\left(v_{1,2^{\circ}}\right)$, imparted to the methyl or ketene products during the dissociation of the vibrationally excited $\mathrm{CH}_{3} \mathrm{C}(\mathrm{O}) \mathrm{CH}_{2}$, and (if necessary) the angular distribution $I\left(\theta_{2^{\circ}}\right)$ using the model described in ref 2 . The new algorithm presented here allows us to also fit the net angular distribution

$$
I\left(\theta_{\text {net }}\right) \propto 1+\beta_{\text {net }} P_{2}\left(\cos \theta_{\text {net }}\right)
$$

where $P_{2}(\mathrm{x})$ is the second Legendre polynomial:

$$
P_{2}(\cos \theta)=\frac{1}{2}\left(3 \cos ^{2} \theta-1\right)
$$

Our new algorithm (included in the Supporting Information) is a continuation of the model described in ref 2 that fit the speed distribution $P\left(v_{\text {net }}\right)$. That program generated an array that stores a sorted list of net speeds of a molecular fragment produced through secondary dissociation, as well as the fraction $P\left(v_{\text {net }}\right)$ that a given range of speeds $v_{\text {net }} \pm \Delta v_{\text {net }} / 2$ contributes to the net speed distribution. In the new algorithm presented here, additional columns of the array record the individual speeds imparted in the primary and secondary dissociation steps, $v_{1,1^{\circ}}$, $v_{1,2^{\circ}}$, and $I\left(\theta_{1^{\circ}}\right)$ values and corresponding probabilities that contributed to a specific $v_{1, \text { net }}$ and $P\left(v_{\text {net }}\right) .^{8}$

Input to the program includes the masses $m_{1}$ and $m_{2}$ produced in the first (photodissociation) step, where $m_{1}$ is the mass of the photofragment that undergoes secondary dissociation, and the distribution of recoil kinetic energies imparted to those initial photoproducts, $P\left(E_{1^{\circ}}\right)$, where $E_{1^{\circ}}=$ $(1 / 2) \mu v_{\text {rel }}^{2}$. These are used to calculate the speeds of the radicals that subsequently dissociate. We also must input the
$\beta\left(E_{1^{\circ}}\right)$ that characterizes the angular distribution of these primary velocity vectors. The Jacobian $\mathrm{d}(\cos \theta)=\sin \theta \mathrm{d} \theta$ is accounted for in the iteration over $\theta_{1^{\circ}} . \beta\left(E_{1^{\circ}}\right)$ is determined from the experimental data taken of the chlorine atom produced in the primary dissociation. The inputted $\beta\left(E_{1^{\circ}}\right)$ must span the same range in energies as $P\left(E_{1^{\circ}}\right)$ as we linearly interpolate between the values provided to allow for small increments in the computed net speeds.

The angular distribution of net velocity vector of the secondary fragment is defined by an angle $\theta_{\text {net }}$ measured with respect to the polarization vector of the laser. From the computed distribution of $\theta_{\text {net }}$ called $I\left(\theta_{\text {net }}\right)$, we determine $\beta\left(v_{\text {net }}\right)$ using eq 3 . Our previous model ${ }^{2}$ only considered the net speed distribution, which is not sensitive to the anisotropy parameter $\beta\left(v_{1^{\circ}}\right)$.

To compute the net angular distribution $I\left(\theta_{\text {net }}\right)$, our algorithm must efficiently treat two coordinate systems with appropriate Euler angle rotations. The laboratory frame is described in the blue coordinate system in Figure 1 where the

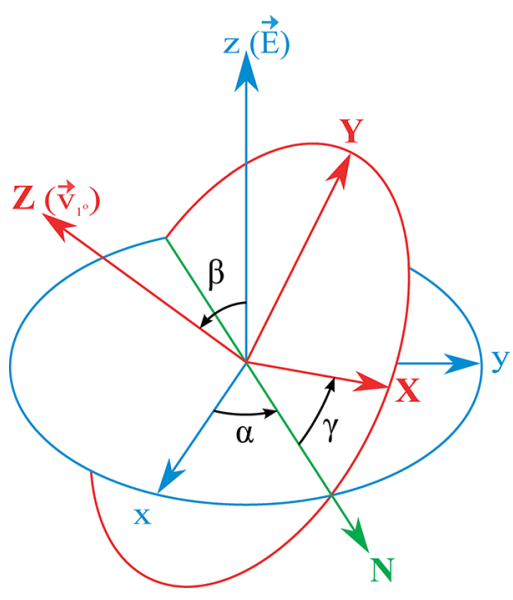

Figure 1. Coordinate systems with Euler angles for the primary photodissociation and subsequent secondary dissociation processes. The blue $z$ axis is parallel to the electric vector of the photodissociation light. The red $Z$ axis lies along the velocity vector imparted to the unstable radical in the primary photodissociation. The axes in the red coordinate system are obtained from the axes in the blue coordinate system using Euler angles as follows: First rotate by $\alpha$ about the blue $\mathrm{z}$ axis, mapping the blue $\mathrm{x}$ axis to the green line of nodes; then rotate by $\beta$ about the green line of nodes, mapping the blue $\mathrm{z}$ axis to the red $\mathrm{Z}$ axis; finally rotate by $\gamma$ about the red $\mathrm{Z}$ axis, mapping the green line of nodes to the red $\mathrm{X}$ axis. (For an animation, see the Wikipedia article on Euler angles under "Euler angles as composition of intrinsic rotations".) The Euler angle $\beta$ is thus the angle $\beta\left(\mathrm{E}_{1^{\circ}}\right)$ between the polarization vector of the laser and the dissociating radical's velocity vector. The Euler angle $\alpha$ corresponds to the azimuthal angle $\phi_{1^{\circ}}$ in the primary photodissociation. The Euler angle $\gamma$ accounts for the azimuthal angle $\phi_{2}$ in the secondary dissociation of the radical. This figure has been adapted from Lionel Brits, Wikimedia Commons, and is used under the Creative Commons Attribution 3.0 Unported License.

laser light travels along the $x$ axis with its polarization vector being aligned with the $z$ axis. If we let the velocity vector $\vec{v}_{1,1^{\circ}}$ imparted to the unstable radical (here, $\mathrm{CH}_{3} \mathrm{C}(\mathrm{O}) \mathrm{CH}_{2}$ ) in the primary photodissociation event lie along the red $Z$ axis in the $X Y Z$ coordinate system, the angle $\theta_{1}$ corresponds to the Euler angle $\beta$ in Figure 1 . The algorithm weights the probability of a particular $\theta_{1}$ o using the primary photofragment angular distribution's anisotropy parameter $\beta\left(v_{1^{\circ}}\right)$ iterating with 
$\Delta \cos \left(\theta_{1^{\circ}}\right)$. The azimuthal angle $\phi_{1^{\circ}}$, the angle by which the relative velocity vector for the $\mathrm{CH}_{3} \mathrm{C}(\mathrm{O}) \mathrm{CH}_{2}+\mathrm{Cl}$ dissociation is rotated out of the plane perpendicular to the direction of travel of the laser light, corresponds to the Euler angle $\alpha$, but it is not necessary to iterate this from 0 to $2 \pi$ as it does not alter the computed $\beta\left(v_{\text {net }}\right)$.

The secondary dissociation of the radical, here the dissociation of $\mathrm{CH}_{3} \mathrm{C}(\mathrm{O}) \mathrm{CH}_{2}$ to $\mathrm{CH}_{3}$ + ketene, is characterized by a secondary recoil kinetic energy distribution, $P\left(E_{2^{\circ}}\right)$, and two angles $\theta_{2}$ and $\phi_{2^{\circ}}$. This secondary recoil is described in the red coordinate system in Figure 1. If a product is "forward scattered", the velocity vector imparted in the secondary dissociation lies parallel to the velocity vector of the dissociating radical, corresponding to a small $\theta_{2}$.

To describe the full 3D angular distribution of this secondary velocity vector, we require two angles. The first is the angle $\theta_{2}$ 。 between the velocity vector of the dissociating radical and the additional velocity vector imparted to the detected fragment during the secondary dissociation, which does not correspond to an Euler angle in Figure 1. Its angular distribution $I\left(\theta_{2^{\circ}}\right)$ is determined by forward convolution fitting of the measured $\beta\left(v_{\text {net }}\right)$. The other angle needed to describe the full 3D angular distribution of the secondary velocity vector is the azimuthal angle $\phi_{2^{\circ}}$. It is uniformly distributed from 0 to $2 \pi$. One may either rotate the secondary velocity vector around the red $Z$ axis in Figure 1, or simply define the secondary dissociation as imparting a velocity in the red $Y Z$ plane and rotate the red coordinate system by the Euler angle $\gamma$ shown in Figure 1 . Thus, our algorithm iterates $\phi_{2}$ 。 from 0 to $2 \pi$ by iterating $\gamma$ from 0 to $2 \pi$, while defining the red $Y Z$ plane as the plane containing the secondary velocity vector. The component of the secondary velocity vector, $\vec{v}_{1,2^{\circ}}$, imparted to the detected fragment along the red $Z$ axis, $v_{z, 1,2^{\circ}}$ (where 1 indicates it is the detected fragment with mass $m_{1,2^{\circ}}$ ), is

$$
v_{z, 1,2^{\circ}}=\left|\vec{v}_{1,2^{\circ}}\right| \cos \theta_{2^{\circ}}
$$

and the component along the red $Y$ axis is

$$
v_{y, 1,2^{\circ}}=\left|\vec{v}_{1,2^{\circ}}\right| \sin \theta_{2^{\circ}}
$$

Given the coordinates above of the net velocity vector in the red coordinate system, we use a rotation matrix $T(\alpha, \beta, \gamma)$ to calculate the coordinates of this vector in the blue coordinate system, defined by the direction of polarization of the laser. The needed transformation is a product of rotation matrices, $T(\alpha, \beta$, $\gamma)=T_{z}(\alpha) T_{x}(\beta) T_{z}(\gamma)$, and is derived in detail in the Supporting Information.

$$
\begin{aligned}
T(\alpha, \beta, \gamma) & =\left(\begin{array}{lll}
\cos \alpha & -\sin \alpha & 0 \\
\sin \alpha & \cos \alpha & 0 \\
0 & 0 & 1
\end{array}\right)\left(\begin{array}{lll}
1 & 0 & 0 \\
0 & \cos \beta & -\sin \beta \\
0 & \sin \beta & \cos \beta
\end{array}\right)\left(\begin{array}{lll}
\cos \gamma & -\sin \gamma & 0 \\
\sin \gamma & \cos \gamma & 0 \\
0 & 0 & 1
\end{array}\right) \\
& =\left(\begin{array}{llll}
\cos \alpha \cos \gamma-\sin \alpha \cos \beta \sin \gamma & -\cos \alpha \sin \gamma-\sin \alpha \cos \beta \cos \gamma & \sin \alpha \sin \beta \\
\sin \alpha \cos \gamma+\cos \alpha \cos \beta \sin \gamma & -\sin \alpha \sin \gamma+\cos \alpha \cos \beta \cos \gamma & -\cos \alpha \sin \beta \\
\sin \beta \sin \gamma & \sin \beta \cos \gamma & \cos \beta
\end{array}\right)
\end{aligned}
$$

We let $\left(v_{x, \text { net }}, v_{y, \text { net }}, v_{z \text {,net }}\right)$ be the coordinates of the net velocity vector $\vec{v}_{\text {net }}$ in the red coordinate system and let $\left(v_{x^{\prime}, \text { net }}, v_{y^{\prime}, \text { net }}, v_{z^{\prime} \text {,net }}\right)$ be the coordinates of the net velocity vector in the lab frame (the blue coordinate system, defined by the direction of propagation and polarization vector of the laser light). The only information needed is the $v_{z^{\prime} \text {, net }}$ component of the net velocity vector in the blue coordinate system in Figure 1 because $v_{z^{\prime}, \text { net }}=\left|\vec{v}_{\text {net }}\right| \cos \theta_{\text {net }}$, where $\left|\vec{v}_{\text {net }}\right|$ is the net speed of the detected fragment (measured in the imaging apparatus). The net velocity vector in the red coordinate system is $\left(v_{x, \text { net }}, v_{y, \text { net }}, v_{z, \text { net }}\right)=\left(0, v_{y, 1,2^{\circ}}, v_{z, 1,2^{\circ}}+v_{z, 1,1^{\circ}}\right)$ where the contribution from the secondary dissociation is given in eqs 5 and 6 and the dissociating radical's velocity is $\left(0,0, v_{z, 1,1^{\circ}}\right)$ in the red coordinate system. $v_{x, 1,2}$ is zero because rotation by the azimuthal angle $\phi_{2}{ }^{\circ}$ in the secondary dissociation is accomplished by iterating the Euler angle $\gamma$. Applying the Euler rotation matrix $T$ to this vector gives the $z$-axis component of the net velocity vector in the blue coordinate system as

$$
v_{z^{\prime}, \text { net }}=v_{y, \text { net }}(\sin \beta \cos \gamma)+v_{z, \text { net }}(\cos \beta)
$$

To calculate $\beta\left(v_{1, \text { net }}\right)$, the program goes through every $v_{1, \text { net }}$ in the sorted array of $v_{1, \text { net }}$ 's and determines $\cos \theta_{\text {net }}$ for that vector using the primary speed $v_{1,1^{\circ}}$, secondary speed $v_{1,2^{\circ}}$, and $\theta_{2^{\circ}}$ that caused a given net speed to occur. There can be several identical net speeds with different $v_{1,1^{\circ}}, v_{1,2^{\circ}}$, and $\theta_{2^{\circ}}$. For a given range of net speeds, the program bins and sums the incidences of the net velocity vector that point in a prespecified $\Delta \cos \left(\theta_{\text {net }}\right)$ range about each $\theta_{\text {net }}$ to obtain $I\left(\theta_{\text {net }}\right)$ and calls the GNU Scientific Library ${ }^{9}$ function gsl_fit_linear to fit the binned $I\left(\theta_{\text {net }}\right)$ points to the functional form $I\left(\theta_{\text {net }}\right)=c_{0}+c_{1} P_{2}$ (cos$\left.\left(\theta_{\text {net }}\right)\right)$. The program does not maintain intermediate normalization because of the prefactor to eq 3 , so $c_{0} \neq 1$, and $c_{1} \neq$ $\beta\left(v_{1, \text { net }}\right)$. However, $c_{1} / c_{0}=\beta\left(v_{1, \text { net }}\right)$. Thus, $\beta\left(v_{1, \text { net }}\right)$ is still accurately determined from this procedure. Additionally, the gsl_fit linear function outputs the residual sum of squares (RSS) for the fit, which is scaled appropriately.

\section{DISCUSSION}

The algorithm described in this paper allows the complete analysis of the measured speed and angular distributions for data taken on a velocity map imaging apparatus for products from a two-step dissociation mechanism. The $P\left(v_{\text {net }}\right)$ and $\beta\left(v_{\text {net }}\right)$ output by BASEX is fit with the algorithm to a two-step model. This allows one to determine the distribution of velocities imparted in the secondary dissociation step, despite the fact that the measured net speed and angular distributions are affected by both dissociations in the two-step process.

The current implementation of the program presumes that the angular distribution of the velocity vectors imparted in the secondary dissociation is independent of the speed imparted to the primary photofragment. However, the code can be easily modified to account for a possible dependence of the secondary angular distribution on the speed imparted to the radical in the 
primary photodissociation event. Additionally, this model can be used to treat tertiary dissociations, those with a primary photodissociation and two subsequent dissociations by first treating the secondary dissociation, and then using the outputted $P\left(v_{\text {net }}\right)$ and $\beta\left(v_{\text {net }}\right)$ as the primary process input and the tertiary process input as the secondary dissociation on the second pass through the program.

In considering what trial forms of $I\left(\theta_{2^{\circ}}\right)$ to use, the standard ones are $\beta=0$ for an isotropic angular distribution of the secondary velocity vector with respect to the primary radical photoproduct's velocity vector or a $1 / \sin \theta_{2^{\circ}}$ distribution to represent the dissociation of a rotationally hot radical with no out-of-plane exit channel forces. However, in some cases, radicals produced by photodissociation with low translational energy (and thus high internal energy) may result in a highly unstable radical that dissociates on a time scale shorter than a rotational period. In this case, the secondary $I(\theta)$ may not be symmetric about $\theta=\pi / 2$. In contrast, radicals produced from photodissociation events that partition more energy to recoil translational energy would have a lower internal energy. These radicals would take longer to dissociate, so their secondary angular distribution might be reasonably modeled by a forward/backward symmetric secondary angular distribution. If the rotational angular momentum of the radicals is also large, the angular distribution of the secondary velocity vectors may be well described by a $1 / \sin \theta$ distribution, discussed by Herschbach ${ }^{10}$ with regard to bimolecular reactions. Examples of this distribution for the secondary dissociation of highly rotationally excited radicals formed from photodissociation of a halogenated precursor can be seen in refs 11 and 12 .

An interesting example of how one might extract physical insight from the secondary angular distribution, derived from a forward convolution fit to the net angular distribution with the code presented here, comes from the dissociation of 2-nitro-2propyl radicals. ${ }^{13}$ Figure 2 shows the $I\left(\theta_{2^{\circ}}\right)$ that characterizes the angular distribution obtained from the secondary dissociation of vibrationally excited $\mathrm{CH}_{3} \mathrm{C}\left(\mathrm{NO}_{2}\right) \mathrm{CH}_{3}$ to $\mathrm{NO}$ $+\mathrm{CH}_{3} \mathrm{C}(\mathrm{O}) \mathrm{CH}_{3}$. (It was derived from fitting scattering data in ref 13 , not the imaging data, as the primary angular distribution

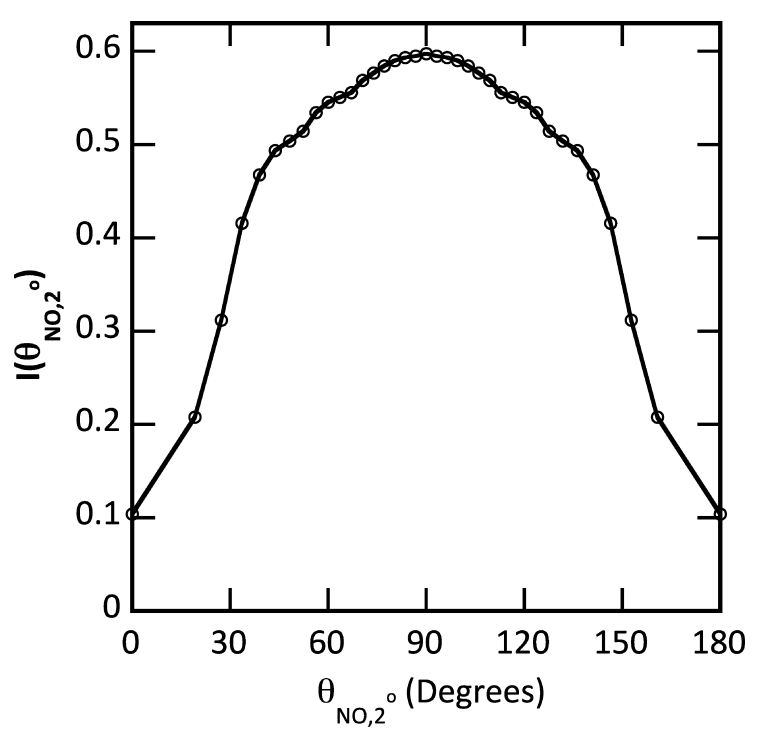

Figure 2. Angular distribution for the secondary dissociation of vibrationally excited $\mathrm{CH}_{3} \mathrm{C}\left(\mathrm{NO}_{2}\right) \mathrm{CH}_{3}$ to $\mathrm{NO}+\mathrm{CH}_{3} \mathrm{C}(\mathrm{O}) \mathrm{CH}_{3}$. Adapted from Figure 18 of ref 13. needed to use the code herein was not measured). This angular distribution shows sideways scattering of the NO product with respect to the primary velocity of the 2-nitro-2-propyl radical. The radical was produced by $\mathrm{C}-\mathrm{Br}$ photofission in 2-bromo-2nitropropane at $193 \mathrm{~nm}$, so the initial $\mathrm{C}-\mathrm{Br}$ fission is from a roughly tetrahedral arrangement of the $\mathrm{C}-\mathrm{Br}, \mathrm{C}-\mathrm{N}$, and two $\mathrm{C}-\mathrm{C}$ bonds in the photolytic precursor. The $193 \mathrm{~nm}$ excitation to a repulsive excited state generates a $\vec{v}_{1,1^{\circ}}$ along the $\mathrm{C}-\mathrm{Br}$ bond direction. The resonance-stabilized 2-nitro-2-propyl radical has a planar geometry of the $\mathrm{NO}_{2}$ group and $\mathrm{C}-\mathrm{N}$ bond, so in the exit channel of the primary photodissociation the radical changes from tetrahedral about the center $\mathrm{C}$ atom to planar. Then en route to dissociation the geometry of the 2nitro-2-propyl radical changes again. In the repulsive region of the secondary dissociation reaction coordinate, the geometry of the radical has changed to one where the $\mathrm{C}-\mathrm{N}$ bond, and thus the direction of the secondary recoil velocity imparted to the NO product, $\vec{v}_{\mathrm{NO}, 2^{\circ}}$, is nearly perpendicular to the velocity vector of the radical from the primary photodissociation. Thus a sideways scattered angular distribution of $\vec{v}_{\mathrm{NO}, 2^{\circ}}$ with respect to $\vec{v}_{1,1^{\circ}}$ results, as shown in Figure 2 . The geometry of the radical in this repulsive region of the secondary dissociation, with the initial velocity vector of the radical from the primary photodissociation $\vec{v}_{1,1^{\circ}}$ superimposed on it, is shown in the Supporting Information of ref 13 .

Important to note is that our model only applies under the assumption that the unstable primary photoproduct dissociates on a millisecond or shorter time scale and does not travel a significant distance before it dissociates. One other consideration is that if using REMPI detection of the atomic coproduct produced in the primary photodissociation, one may need to use higher order Legendre polynomials to fit the measured angular distribution if $J>1 / 2:^{14}$

$$
I(\theta, \phi) \propto 1+\beta_{2} P_{2}(\cos \theta)+\beta_{4} P_{4}(\cos \theta)
$$

In summary, we have introduced a new computational program for fitting net angular distributions from two-step photodissociation mechanisms in velocity map imaging, and this program allows us to test whether a particular distribution of velocity vectors imparted in the secondary dissociation is consistent with the recorded anisotropy data.

\section{ASSOCIATED CONTENT}

\section{S Supporting Information}

The code for our algorithm, analysis of chloroacetone experimental data, and a detailed derivation of eq 8 . This material is available free of charge via the Internet at http:// pubs.acs.org/.

\section{AUTHOR INFORMATION}

\section{Corresponding Author}

*E-mail: L-Butler@uchicago.edu.

\section{Present Addresses}

${ }^{\S}$ Department of Chemistry, University of Pennsylvania, Philadelphia, PA.

"Department of Chemistry, Emory University, Atlanta, GA.

\section{Notes}

The authors declare no competing financial interest.

\section{ACKNOWLEDGMENTS}

This work was supported by the National Science Foundation (NSF) under Grant No. CHE-1152043. We thank M. Hillegass 
for calculating the energetics of the dissociation channels of the $\mathrm{C}(\mathrm{O}) \mathrm{CH}_{2} \mathrm{Cl}$ radical.

\section{REFERENCES}

(1) Zhao, X. CMLAB2, version 6/93; modified by J. D. Myers. This is an interactive version built on the original cmlab2 program: Zhao, X.; Ph.D. dissertation, University of California, 1988.

(2) Alligood, B. W.; Straus, D. B.; Butler, L. J. Analyzing Velocity Map Images to Distinguish the Primary Methyl Photofragments From Those Produced Upon $\mathrm{C}-\mathrm{Cl}$ Bond Photofission in Chloroacetone at 193 nm. J. Chem. Phys. 2011, 135, 034302.

(3) Martinez-Haya, B.; Bass, M. J.; Brouard, M.; Vallance, C.; Torres, I.; Barr, J. Photodissociation and Multiphoton Dissociative Ionization Processes in $\mathrm{CH}_{3} \mathrm{~S}_{2} \mathrm{CH}_{3}$ at $193 \mathrm{~nm}$ Studied Using Velocity-Map Imaging. J. Chem. Phys. 2004, 120, 11042-11052.

(4) Alligood, B. W.; FitzPatrick, B. L.; Szpunar, D. E.; Butler, L. J. Chloroacetone Photodissociation at $193 \mathrm{~nm}$ and the Subsequent Dynamics of the $\mathrm{CH}_{3} \mathrm{C}(\mathrm{O}) \mathrm{CH}_{2}$ Radical-An Intermediate Formed in the $\mathrm{OH}+$ Allene Reaction en Route to $\mathrm{CH}_{3}+$ Ketene. J. Chem. Phys. 2011, 134, 054301.

(5) Chang, B.; Hoetzlein, R. C.; Mueller, J. A.; Geiser, J. D.; Houston, P. L. Improved Two-Dimensional Product Imaging: The Real-Time Ion-Counting Method. Rev. Sci. Instrum. 1998, 69, 1665-1670.

(6) Dribinski, V.; Ossadtchi, A.; Mandelshtam, V. A.; Reisler, H. Reconstruction of Abel-Transformable Images: The Gaussian Basis-Set Expansion Abel Transform Method. Rev. Sci. Instrum. 2002, 73, 26342642.

(7) Zare, R. N. Angular Momentum: Understanding Spatial Aspects in Chemistry and Physics; Wiley: New York, 1988.

(8) Note that $I\left(\theta_{2^{\circ}}\right)$ is simply referred to as $I(\theta)$ in ref 2. As it describes the secondary dissociation, not the net angular distribution, it is defined in reference to the recoil velocity vector of the $\mathrm{Cl}$ and $\mathrm{CH}_{3} \mathrm{C}(\mathrm{O}) \mathrm{CH}_{2}$ radicals and is distinct from $I\left(\theta_{1^{\circ}}\right) . I\left(\theta_{1^{\circ}}\right)$, which defines the primary photofragment angular distribution, is obtained by detecting chlorine atom images and input to the program by specifying $\beta\left(E_{1^{\circ}}\right)$ in conjunction with the portion of the primary photofragment $P\left(E_{1^{\circ}}\right)$ that results in dissociating radicals.

(9) Galassi, M.; Davies, J.; Theiler, J.; Gough, B.; Jungman, G.; Alken, P.; Booth, M.; Rossi, F. GNU Scientific Library: Reference Manual for GSL Version 1.12; Network Theory: Bristol, U.K., 2009.

(10) Herschbach, D. R. Reactive Collisions in Crossed Molecular Beams. Faraday Discuss. 1962, 33, 149-161.

(11) Ratliff, B. J.; Alligood, B. W.; Butler, L. J.; Lee, S.-H.; Lin, J. J.-M. Product Branching from the $\mathrm{CH}_{2} \mathrm{OH}$ Radical Intermediate of the $\mathrm{OH}$ + Ethene Reaction. J. Phys. Chem. A 2011, 115, 9097-9110.

(12) Hintsa, E. J.; Zhao, X.; Lee, Y. T. Photodissociation of 2Bromoethanol and 2-Chloroethanol at $193 \mathrm{~nm}$. J. Chem. Phys. 1990, 92, 2280-2286.

(13) Booth, R. S.; Lam, C.-S.; Brynteson, M. D.; Wang, L.; Butler, L. J. Elucidating the Decomposition Mechanism of Energetic Materials with Geminal Dinitro Groups Using 2-Bromo-2-Nitropropane Photodissociation. J. Phys. Chem. A 2013, DOI: 10.1021/jp312248v.

(14) Rakitzis, T. P.; van den Brom, A. J.; Janssen, M. H. Molecular and Laboratory Frame Photofragment Angular Distributions from Oriented and Aligned Molecules. Chem. Phys. Lett. 2003, 372, 187194.

\section{NOTE ADDED AFTER ASAP PUBLICATION}

This article posted ASAP on March 6, 2013. In the Discussion section, paragraph 4, sentences 9 and 10 have been revised. The correct version posted on March 8, 2013. 